\title{
Enhanced MQTT Protocol by Smart Gateway
}

\author{
Hawraa R. Abdul Ameer ${ }^{1}$,Hamid M. Hasan ${ }^{2}$ \\ Control and Systems Engineering Department, University of Technology, Baghdad-Iraq \\ Control and Systems Engineering Department, University of Technology, Baghdad-Iraq \\ HUC.hawraa.alameer@gmail.com, hamid2012net@gmail.com
}

\begin{abstract}
The MQTT protocol is one of the Internet of Things (loT) application protocols. It is based on publishing/subscribing messaging patterns. This protocol uses a broker to manage the communication between the subscribers and the publishers over the internet. There were two problems associated with the implementation of this protocol. The first one, there are many sensors generating huge data using the very simple IoT architecture. These big data may lead to congestion on the internet and thus will cause several disadvantages such as decreasing throughput, packet loss, and delay, which in turn may affect the performance of the MQTT broker, thus affecting the mechanism of the performance of the IoT application. The second problem, the MQTT protocol does not contain a priority management algorithm which is considered a very important and special issue in health applications. In this paper, a smart gateway is designed to enhance the MQTT networks. This work introduced the smart e-health gateway used in MQTT based remote Electrocardiogram (ECG) monitoring system. A new architecture has been implemented that contains two new components, the local broker and the publisher /subscriber (pub/sub) models. The local broker works in the local network and the publisher /subscriber (pub/sub) model is responsible for the process of smart in the gateway. One of the smart applications was used is a filter application. We've taken the data filtering side and give it a priority, as an example in order to know the possibility of achieving the architecture of the gateway that was created in our work. And is this architecture valid to work or invalid. Therefore, we conducted a test using the application of ECG signal filtration and give priority to patients according to the case of an urgent for each patient. The R-peaks were calculated from the ECG signals-peaks for the identification of heart rate. The urgent level will be classified depending on the beats rate feature. The proposed smart gateway function is to manage the data rate that will pass through to the internet. In the case of high urgent level, all the packets will be passed, while in case of a low level, the least possible data will be passed. The proposed system scenarios are designed and evaluated by using the OMNET simulation environment and Inet 4 framework. The simulation results are shown by the evaluation methods used (delay, throughput, packets sent, and packets received). These results proved the success of the architectural work that was implemented in the system.
\end{abstract}

Index Terms - IoT, Smart Gateway, MQTT, e-Healthcare, ECG, OMNET++, INET.

\section{INTRODUCTION}

The Internet of Things (IoT) is an imaginative and famous idea that has presented another vision of the internet in better productivity, accuracy, and economic advantages. This idea was proposed by Kevin Asheton in 1999. The communication arrangement of IoT utilizes wireless sensor networks (WSNs) to associate the physical world to the internet. The design of IoT depends on specific segments as embedded gadgets with low power and short-run innovations. These gadgets work WSNs as omnipresent availability. IoT will be connected 
in various zones, for example, energy, health, smart cities, home automation, environment protection, etc. [1]. The present transformation in the internet, portable, and machine-tomachine (M2M) innovations can be viewed as the principal period of the IoT [2]. In the coming years, the IoT is required to connect different advancements to empower new applications by associating physical items together with the help of clever decision making. The utilization of IoT expects access to information created by sensors progressively according to their needs. One of the significant ingredients that are essential to achieve the IoT are the gateways. Gateways are intended to interface the sensor/ actuator space to the application area. Gateways total the continuous sensor information powerfully and afterward this information is imparted to the applications that need it over the internet. For instance, a healthcare/mobile medical gateway accumulates information from various sensors conveyed for blood sugar, blood pressure, heart rate, and so on. This information is then totaled, filtered, broken down and lastly sent to the applications that need them [3]. Many protocols have been proposed in this specific situation and those of the user layer have a major prevalence. The most utilized convention for IoT application layer is the MQTT which is intended to ensure the great correspondence of low power and constrained gadgets. But the MQTT protocol application faces several problems, including that it uses TCP / IP and it is very complex for low power, simple devices such as wireless sensoractuator (SA) devices. The other problem is that the MQTT protocol does not have a priority management algorithm which is a very important issue, especially in health applications. In order to overcome these problems and improve the work of MQTT, we have added an algorithm for the gateway to help solve these problems. In this paper, a proposal was made to prioritize the data in the remote transport gateway of the message queue (for sensor networks) in order to mitigate the delay of packet data necessary for timesensitive applications. Because there are hundreds and thousands of sensors scattered in the sensor fields, all these sensors will pass the collected information through them to the MQTT gateway to send this information to the respective applications in the cloud. A smart gateway is designed to enhance MQTT networks. A very important aspect of the Internet of Things (IoT) application is the smart e-health gateway used in the MQTT-based remote Electrocardiogram (ECG) monitoring system. Much related work has been done on many issues related to improve IoT protocols in e-health applications and in other areas, for example:

S. Guoqiang, et al. (2013) [4] proposed a smart internet of things gateway that gives important benefits. They used the mobile application processor for the gateway main controller. P. Santos, et al. (2015) [5] proposed a structure that allows remote control of a smart environment (home with various sensors and network-connected objects) and to support and implement learning algorithms and understand the user routine so that it reduces tasks that are less importance. They used Raspberry Pi as a gateway to run Node and to communicate with other sensors. Y. Hang and S. Nair, (2016) [6] described a lightweight application of the IoT gateway framework where you can use build applications that include sensor and engine information, and the event base and processing. P. Karhula, (2016) [7] presented an application of the IoT gateway, which allows Internet connectivity to many different peripherals using protocols designed for the IoTs. Raspberry Pi 2 was chosen as the gateway computing platform. Z. Yang, et al. (2016) [8] proposed a new method of monitoring ECG based on IoT technologies. ECG data were collected using a wearable monitoring node and sent directly to the Internet of Things cloud using Wi-Fi. HTTP and MQTT were used in the IoT cloud. A. Rahman, et al. (2018) [9] introduced the idea of fog detection and smart e-health gateways in relation to the IoT based social security 
framework. They used in the health field to measure and analyze the ECG signal. A. Mishra, et al. (2018) [10] proposed another strategy for ECG observing depending on lightweight MQTT keeping IoT at the top of the priority list. M. Ngo, et al. (2018) [11] presented two case studies that were used, the first using automated learning to detect user methods and the second on unloading tasks by designing an algorithm for a smart device. They used Raspberry-Pi 3 as a gateway. S. Kenitar, et al. (2018) [12] gave us an opportunity to study and evaluate the famous latency MQTT protocol, in the industrial field. It allows the user to publish/subscribe to his structure by designing the intercontinental time test. This experiment also tests the latency on different gateways of the MQTT.

The objective of this paper can be divided into four steps:

1- Selecting the IoT based health care architecture as a field for this paper.

2- Simulating the network architecture using OMNET++ and evaluating the performance in terms of throughput and end to end delay etc.

3- Adding data reduction algorithms into the MQTT based gateway and intelligent algorithm(s) to achieve the required priority to enhance the MQTT protocol.

4- Simulating the new design using OMNET++ and evaluating the performance of the new design and then comparing with outcomes in step 2.

This paper is arranged as follows: Section 2 explains the background. Section 3 explains the tools and methodology. Section 4 discusses the implementation and results. Section 5 gives the conclusions of the proposed system.

\section{BACKGROUND}

\section{A. Internet of Things (IoT)}

Internet of things systems have general architecture and a set of common elements. These common elements include physical objects (equipped with various technologies, actuators, and sensors), network infrastructure (routers, switches, gateways, etc.), cloud computing entities (scalable computing storage and power, etc.) and various interfaces for end users and other systems. The elements can be additional organized in architectural models as observed in most studies above. They have these patterns variation in their accuracy but the main concept between them is generally consistent. The basic system architecture of IoT it is clear in Figure (1)

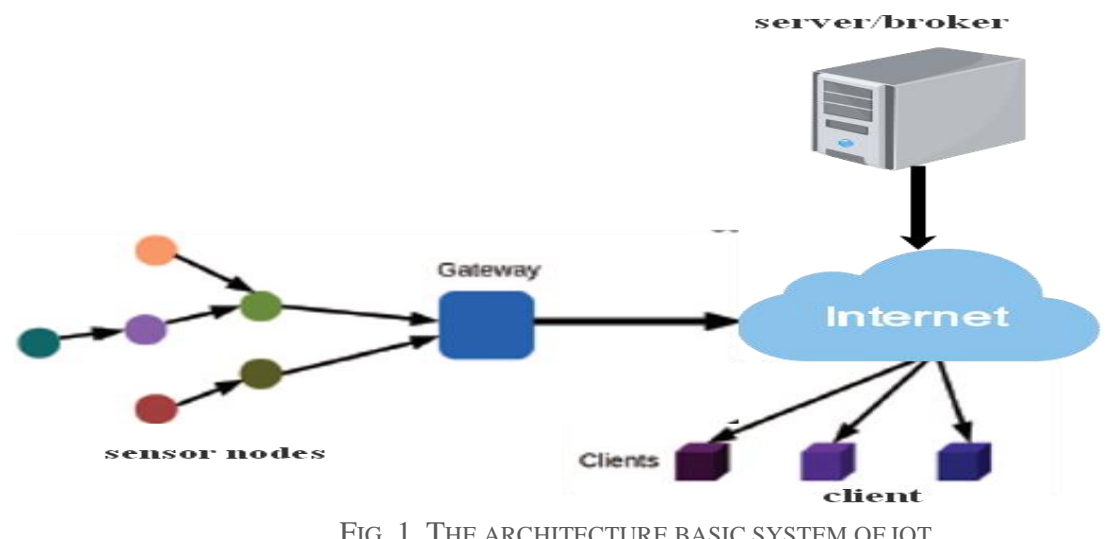

\section{B. IoT Protocols}

Defining the protocol design for the actuator/sensor interconnection is a significant challenge in IoTs. IEEE develops a new communication protocol that can function as a major fraction of the execution of 
IoT applications. Low energy sensors and small rate communications are the primary barriers while developing an IoT protocol. In IoT, after converting and filtering, equipment and sensors retrieve the information and send it to the server. The server analyzes and shares the data with machines or individuals [11]. For IoT, several protocols used such as MQTT, AMQP, XMPP, and COAP, are accessible, and MQTT is most frequently used. MQTT's main characteristic is its lightweight and effectiveness in bandwidth [18]. The next passage will describe the MQTT, which is the most frequently utilized protocol in IoT.

\section{MQTT Protocol}

MQTT is an abbreviation of ("Message Queuing Telemetry Transport") which is a protocol operating over the TCP / stack application level [11]. The protocol that uses client-server using a subscribe-publish messaging sample is plain lightweight and plain to implement. The protocol's reliable and fashionable state makes it best to use it in contexts such as Machine to Machine (M2M) or IoT where a tiny code footprint is needed [13].

\section{Gateway}

In the network, the gateway is a hub on a TCP/IP organize that fills in as a passage to another system. In the field of IoT and savvy building/lodging, the gateway gives the extension between gadgets in the physical world, the cloud, where information is gathered, put away and controlled by big business applications and the client gear. The smart gateway basically has four tasks: (1) configuration and management of devices; (2) filtering and handling of data; (3) temporary storage of information; and (4) extra input of information [14].

\section{E. IoT in the Health Domain}

IoT will assume a basic job to create astute administrations for supporting and improving individuals' and societies' exercises. Overall population medicinal services are a significant issue given the exponential ascent in the development of the populace and medical costs nowadays. Healthcare is one of the IoT innovation's fastest increasing application regions one of the internet's most significant benefits is that we can monitor patient health remotely.

\section{TOOLS AND METHODOLOG}

\section{A. The system's Architecture}

Figure 2 shows the architecture of the internet of thing in the field of e-health.

Our proposed system in this paper was to measure and monitor the ECG signal by using a lightweight MQTT protocol. The system structure of propose design frame is divided into four main parts: the sensor node (publishers), the gateway, the internet cloud (MQTT) and the client (subscribers). These parts will be explained in the following steps:

\section{1- The Sensor Node (Publisher)}

The first aspect of propose design is the network sensor. It is used to connect wireless communications such as personal area networks (PAN) and wireless (WI-FI) to body area network (BAN). BAN is a sensor node connected to people's objects and provides sensor information inside the body. PAN is a set of fixed-position sensor nodes with multiple sensors providing relative temperature and humidity, light sensors, microphone, camera and so on. In our design, we used a set of sensors that we attached to the body of the individual or patient. The sensor part of the ECG sensor is used to detect heart rate in people using pulse sensors even when the individual or patient is at home / hospital. The sensor is then connected to the gateway. 


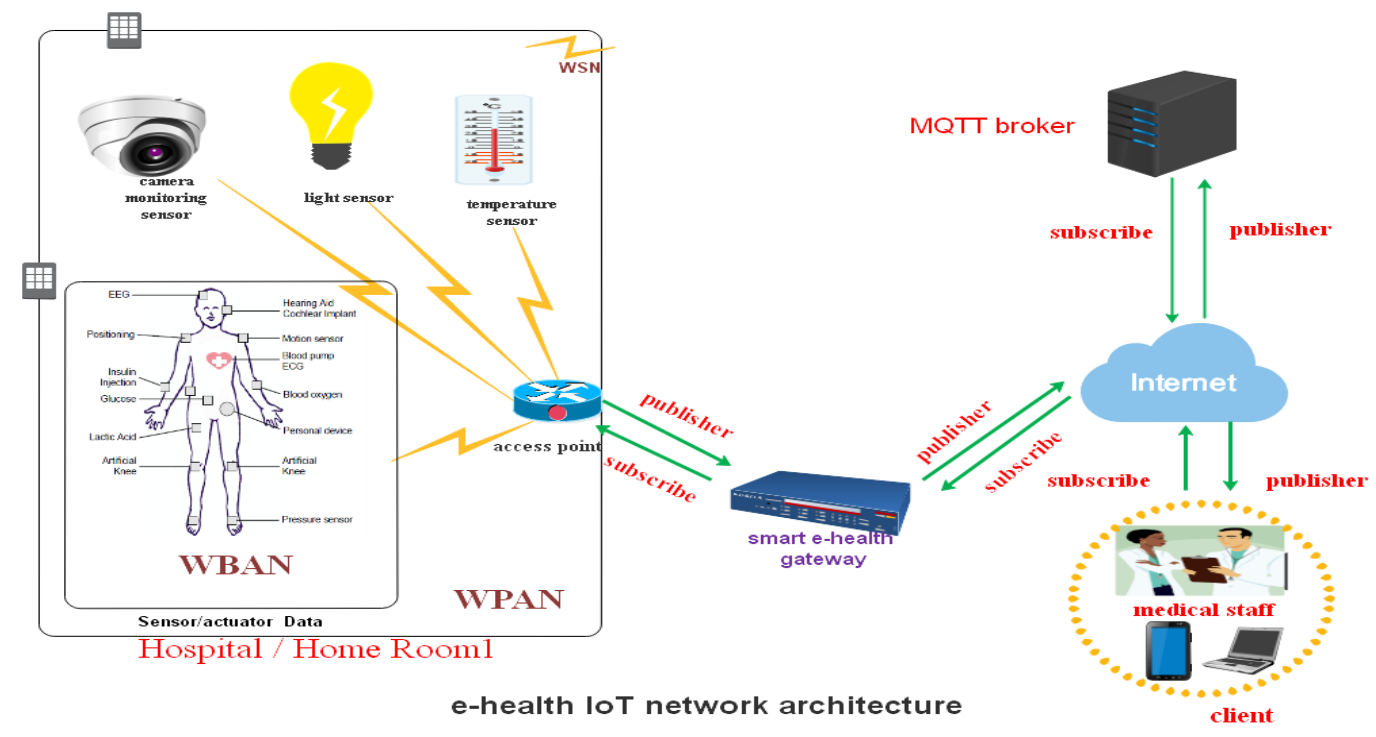

FIG. 2. THE E-HEALTH IOT NETWORK ARCHITECTURE OF THE SYSTEM.

\section{2- Cloud Internet (Broker MQTT)}

The MQTT broker is part of cloud, a business that receives the signal from the publisher and sends it to the subscriber, and can be more than one subscriber on the same signal and one subscriber can take more than one patient. In propose design, eight subscribers were used, namely reception.

\section{3- The Client (Subscriber)}

Common is an application module for TCP clients, similar to the publisher. The client waits for any signal sensor to be automatically sent from the publisher when the signal is reached by the broker. In our design, the client is either a doctor or a nurse, each with the authority to enter and disclose the patient's specific information through MQTT protocol security by password and user name. The customer has one or more subscribers.

\section{4- Gateway Architecture}

The gateway architecture was constructed and that consisted of two main components: the local broker and the publisher/subscriber (Pub/Sub) models as shown in Figure 3. The function of this architecture is to do the fog computing process by processing local data coming from WSN before moving to the cloud.

\section{- Local Broker Model}

The mechanism of action of the local broker is very similar to the work of the cloud broker, which is described earlier in Section (A). But its work limited only on the local network not instead of the cloud. It is directly connected to the sensor side by WSN and the other side is connected to the publisher/subscriber model.

\section{- Publisher / Subscriber (Pub/Sub) Model}

The Pub/Sub model is the main component and important in the construction of the gateway architecture and the importance of adding this model, highlights in conduct processing for 
data on any smart application before sending it. The principle of operation of this model is based on fog Computing by processing the data coming from one side and then sent to another. The mechanism of operation of this model as shown in Figure (3), this is done by sending the signal from the publisher (sensor) to the local broker, where the local broker sends the data to the Pub/Sub model. When the signal arrives at the Pub/Sub model, it performs the necessary processing for data and then publish it to the cloud broker.

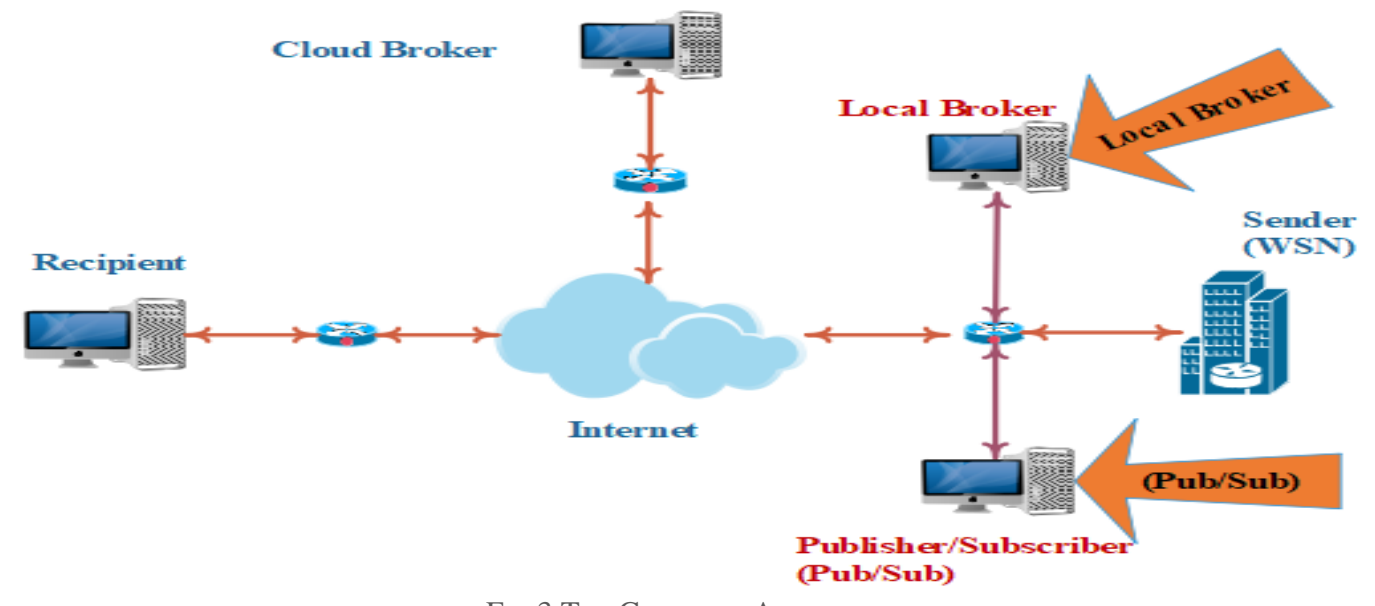

Fig.3.The Gateway Architecture.

\section{Smart Apps}

The benefit of this smart gateway architecture is, where the Pub/Sub model is the one who adopts the work of smart by creating an application that has been called the smart apps. This application can contain many aspects of the smart and the most important:

- Data compression.

- Artificial intelligence (Neural Network, Genetic Algorithm, etc.).

- Data filtering.

The data filtering side has been taken and given it a priority from these many aspects, as an example in order to achieve the validation of designed architecture of the gateway. And for that, we conducted a test using the application of ECG signal filtration and give priority to patients according to the case of an urgent for each patient. The application and measurement of the heart signal will be explained in detail in sections $(\mathrm{B}(2)$ and $\mathrm{D})$.

\section{A. The Network Model.}

This section can be divided into two models: gateway model and smart gateway model.

\section{1- Gateway Model}

The implementation scenario includes a subscriber, publishers, and one broker. These main sections are connected to the cloud internet module. We used the MQTT model, which was recently developed and described in reference [15]. Figure 4 below illustrates the proposed design of Scenario 1 in OMNET, which consists of sensors, gateway, actuators and the MQTT server, which symbolizes the broker. These parts are explained in details in section (A). 


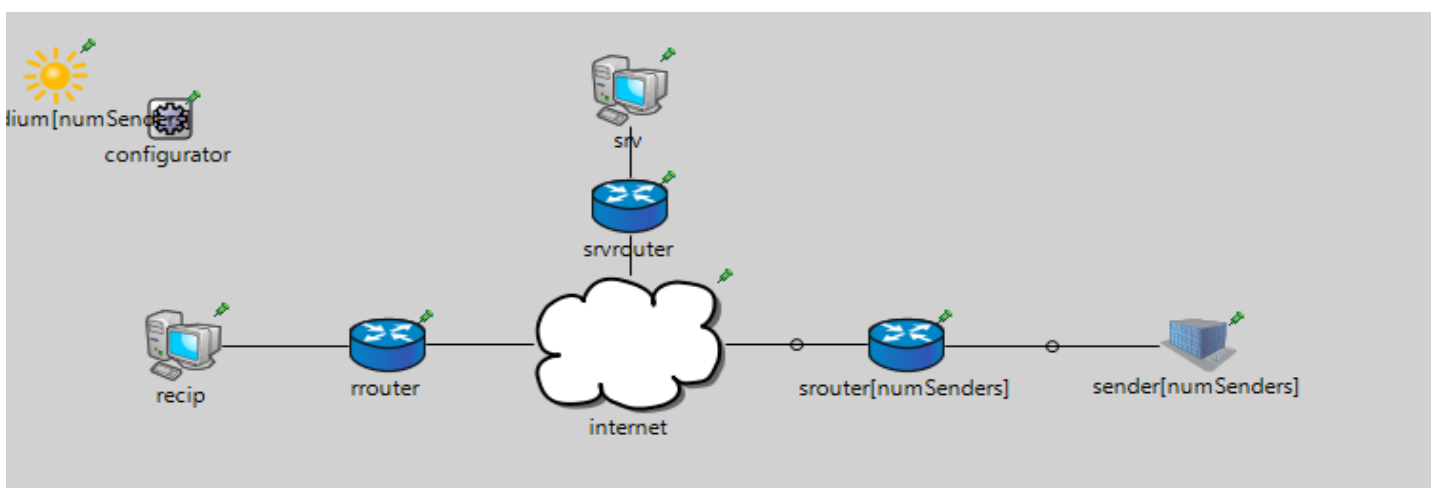

FIG.4.THE PROPOSED DESIGN OF MQTT PROTOCOL.

In Scenario 1, the process of sending data from the sender to the receiver will be according to Table 1 [11], where in the table we note that there are three values for the sample rate, including the normal urgent situation (64 HZ), medium urgent (128 HZ) and high urgent $(256 \mathrm{HZ})$, where the highest value in 256 cases is very serious or called a critical condition. In the case of non-use of the smart gateway, in the normal case of the gateway, we must make the system cover all the situations that exist and this is done by running the rate of sensors with high accuracy for data transmission.

Table 1. State Profiles with Sampling Rate f(HZ) [11].

\begin{tabular}{lc}
\hline State profile & $\begin{array}{c}\text { ECG } \\
\text { frequency }\end{array}$ \\
\hline Normal & 64 \\
Urgent-medium & 128 \\
Urgent-high & 256 \\
\hline
\end{tabular}

Eight nodes were used in the work, each node contains several sensors. The nodes are divided into packets. Each packet is divided into four signals. Each signal of the size of the rate is (46) sample rate, which represents the normal state of the patient and the total size of the four signals per packet is (256) sample rate. In the normal case, only one signal is sent and the other three signals are dropped for each packet. In the case of high urgent, the four signals are sent completely for each packet. We send the highest value of rate. The higher the rate is, the higher the accuracy of the transmitter. This is very important in order to reveal critical situations.

\section{2- Smart Gateway Model}

In this section, we will explain and discuss the methods used to design the smart gateway in detail. Figure 5 below illustrates the proposed design of the smart gateway for Scenario 2 in OMNET ++. Cenario 2 contains four parts: publishers, subscribers, cloud/broker, and smart gateway. The smart gateway design is implemented by adding two standard hosts (Pub/Sub) with the local broker to the previous design. In the case of the smart gateway and through Scenario 2, we made all the sensors work at the highest value of the sample rate (256 HZ). We distributed the load and delay on eight relative cases which are distributed to the number of nodes. In the first case, we note that the high urgent nodes number ratio is only $12.5 \%$ of total sensor nodes, which sends at signals rate of (256) signal per sec, while the rest sends at signals rate of (64) signal per sec, then working on the same mechanism until we reach the eighth and final situation percentage of (100\%). In this case, the smart gateway is similar to the original signal rate at the normal gateway. In the case of the smart gateway, we can 
work with these ratios, so we can reduce delays and load data while in the absence of a smart gateway we cannot do so. The algorithm that we have built in our work for the smart gateway and its working mechanism are illustrated in the following flowchart shown in Figure 6.

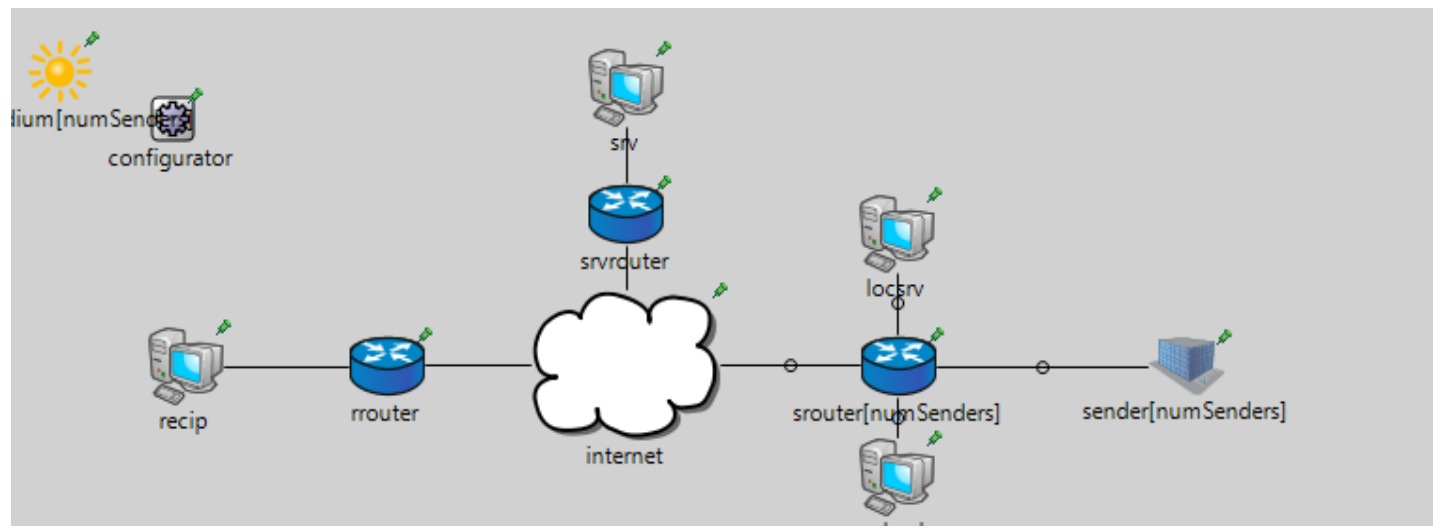

FIG.5.THE PROPOSED DESIGN OF THE SMART GATEWAY IN THE MQTT PROTOCOL

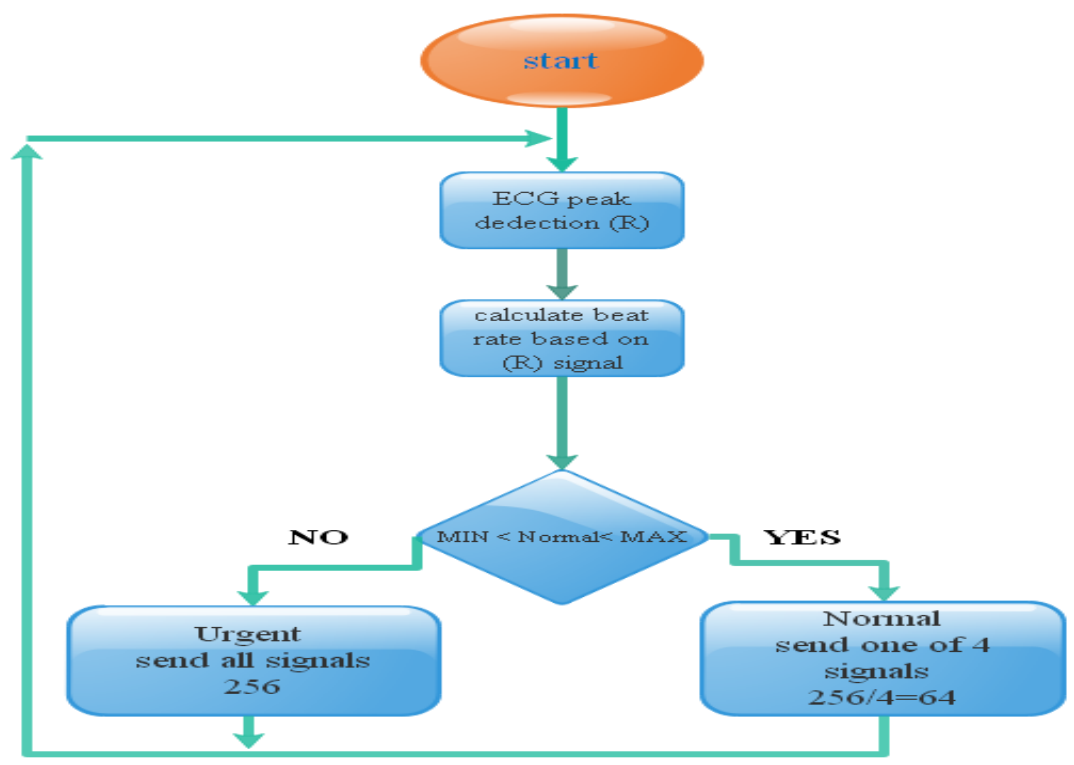

Fig.6. THE FLOWCHART OF SMART GATEWAY.

The smart in this model depends on the beat rate of the heart signal. The method used to extract heartbeat rate is illustrated in Figure 7 below [17].

In general, the heart rate of the patient is supposed to be normal if it is in the range (60-100), while if it is less than 60 or more than 100, it becomes urgent which becomes a critical condition.According to these ranges, the smart gateway will classify the urgent level cases [11], [7]. The smart gateway works as follows: If the condition is normal, only one of the four signals in the packet should be transmitted while the other three signals are ignored. In the urgent case, the smart gateway will transmit the complete signal packet to the broker, so that the client can identify critical cases. 


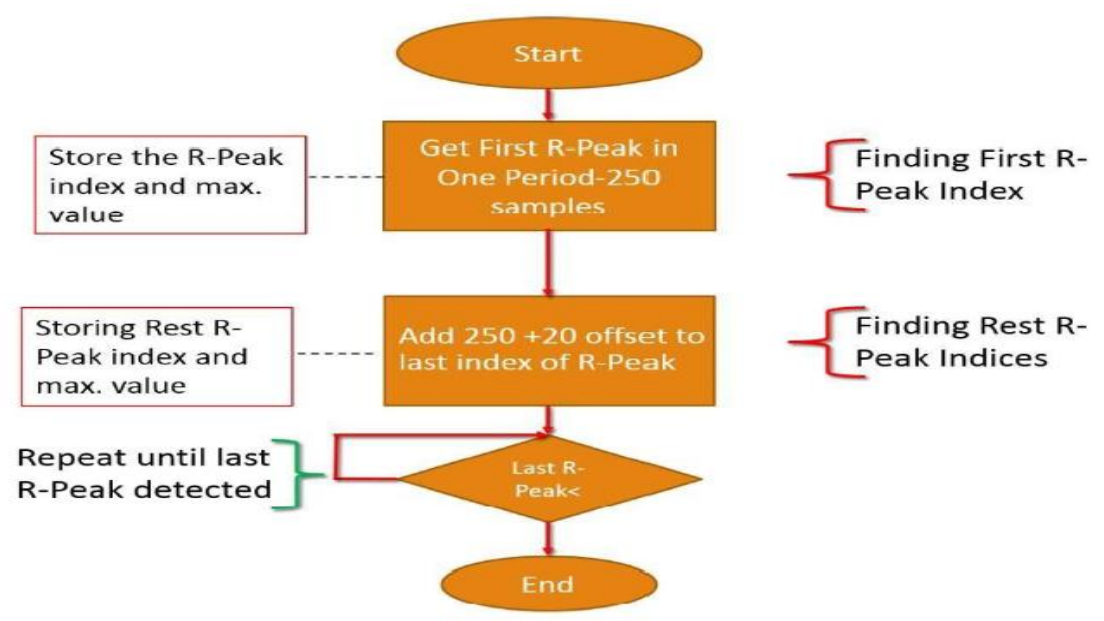

Fig.7. THE FLOWCHART OF BEAT COUNTING [17].

\section{B. Gateway Architecture Programming Modules}

This part is divided into two parts: The local broker programming module and the publisher/subscriber (Pub/Sub) programming module.

- Local Broker Programming Module

The mechanism of action of the local broker is quite similar to the work of the cloud broker.

\section{- Publisher/Subscriber (Pub/Sub) Programming Module}

The function of the publisher/subscriber programming is doing publish and subscribe at the same time, this is done by connecting as a subscriber with the local broker and at the same time connect to the cloud broker as a publisher. The mechanism of the work of this model is subscription to the broker and then receive the signal from the publisher (sensor) and then processing this signal. The processing is based on the smart application that was used.After processing the signal it will publish to the cloud broker. Figure 8 shows a flowchart for both local broker and publisher/subscriber.

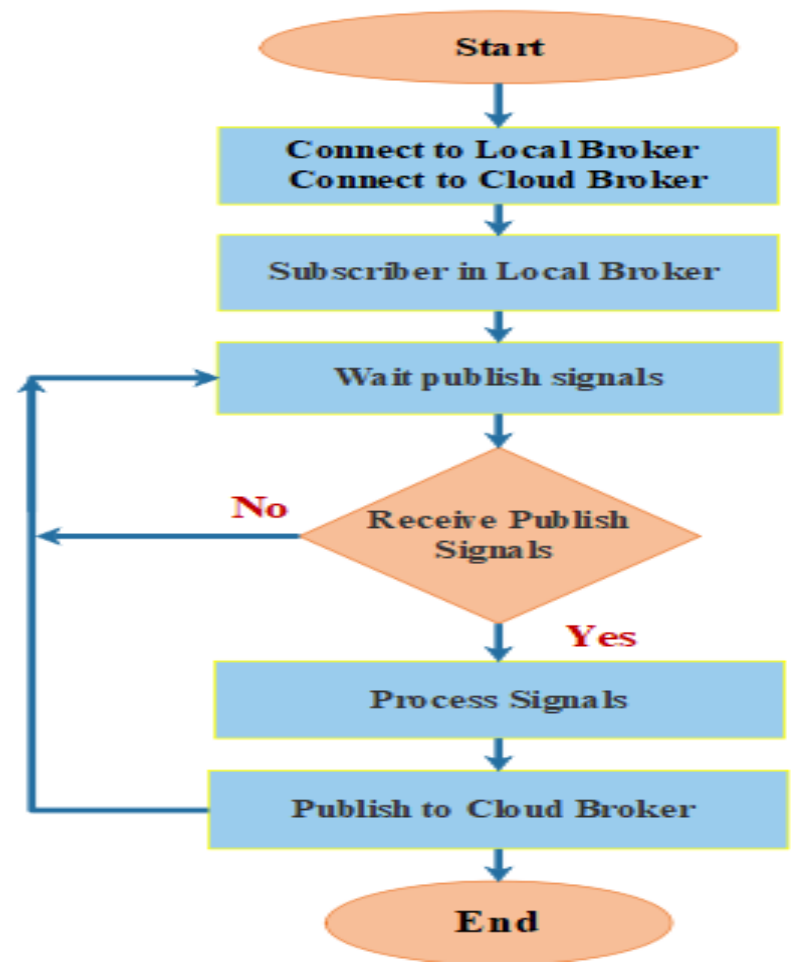

Fig.8. The Flowchart of the Gateway Architecture Programming Modules. 


\section{ECG Packet Design}

To ensure the process of sending and receiving heart rate signals accurately, a function simulation ECG [16], which is built with the MATLAB, was used to generate a heart rate signal as shown in Figure 9.

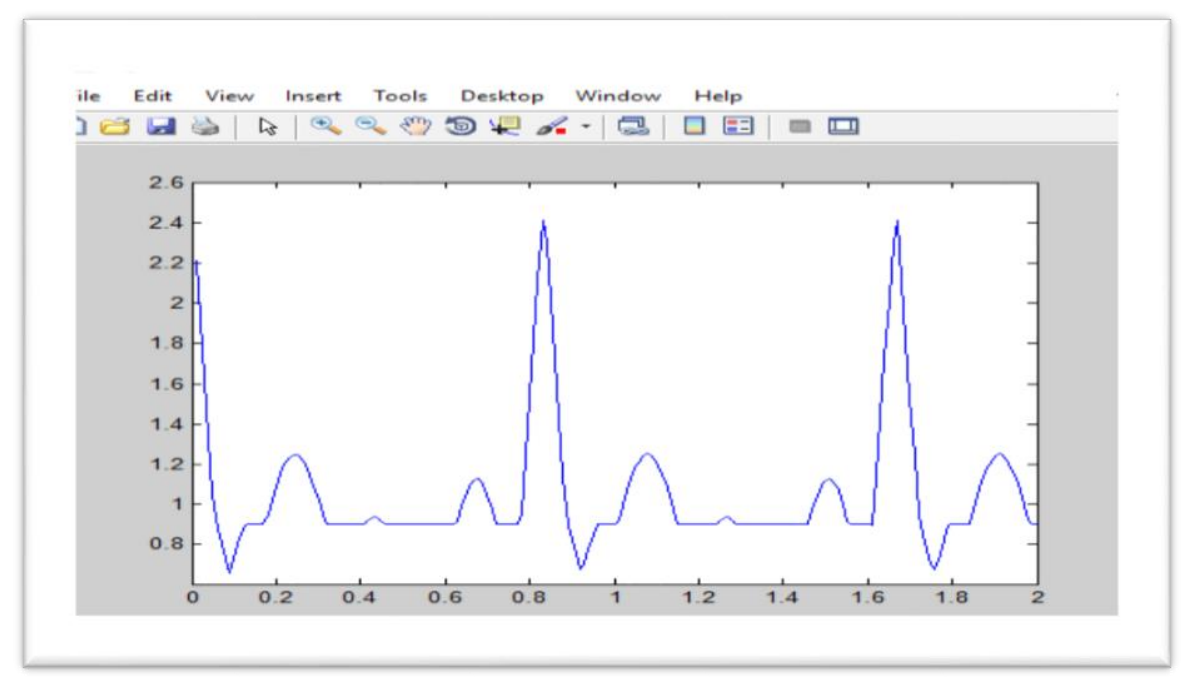

Fig.9. ECG Signal INPUT THAT WAS GENERATED IN THE SimUlation MATLAB.

The generated signals then had been used in the simulation environment of OMNET++ as real data buggy backed in the payload field of the MQTT packets. The following steps were implemented to get the final results:

1- The MATLAB function was modified to convert values of float numbers to binary then to an integer. This procedure is to simplify the work in OMNET++ by using integer numbers.

2- Then the integer data signals were copied to XML. file, which was linked to the .INI file in the OMNET, where the OM sends and receives these signals in the form of a queue and the mechanism of work (FIFO) of the signals.

3- At the publisher model, the number of signals can be controlled by the parameter named "sigPerPacket", the value that was used in this work was 5 ECG signals per one packet as shown in the following INI statement: Sender [*]. sensor [*]. app [0]. *sigPerPack = 5

Each signal was paired with a sequence number to ensure the time location of it. The resolution of each one of these two values has been appointed as 32 bit. In this case, the overall size of the signal will be 64 bit or 8 bytes. By multiplying the 8 bytes by 5 the payload size will be 40 bytes for each packet.

4- The time of the packet being sent based on the sampling rate of the ECG [15] that has been calculated as shown below.

At the frequency $(64)==1 / 64 * 5=0.0781 \mathrm{sec}$

At the frequency $(128)==1 / 128 * 5=0.039 \mathrm{sec}$

At the frequency $(256)==1 / 256 * 5=0.019 \mathrm{sec}$

5- When all the processes of our experience in the OMNET program are completed and after showing the results we transferred the results and stored in the Excel sheet, which is attached to the program code in MATLAB to convert the signal from the output to the original value again and then read and draw the resulting ECG signal as shown in Figure 10. 


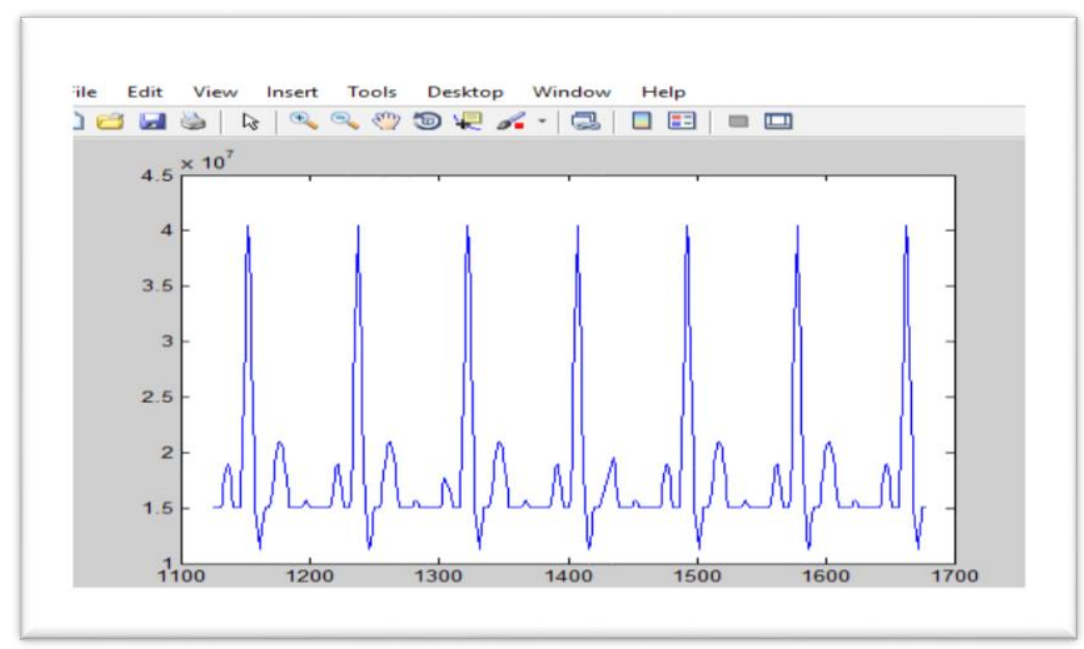

Fig.10. ECG Signal Output that was GENERATEd in the Simulation MATLAB.

\section{Modules Parameters}

Parameters of the sensor node and recipient node module are configured in INI.file, as shown in Table (2) \& Table (3):

TABLE 2. THE SENSOR NODE PARAMETERS

\begin{tabular}{lc}
\hline Parameters name & value \\
\hline local Port & -1 \\
connect Port & 80 \\
start Time & $3 \mathrm{~s}$ \\
request Length & $20 \mathrm{~B}$ \\
reply Length & exponential(20B) \\
header Length & 12 \\
sensor*.numApps & 1
\end{tabular}

TABLE 3. THE RECIPE NODE PARAMETERS

\begin{tabular}{lc}
\hline Parameters name & value \\
\hline local Port & -1 \\
connect Port & 80 \\
start Time & $1 \mathrm{~s}$ \\
request Length & $20 \mathrm{~B}$ \\
reply Length & exponential(10B) \\
recip.numApps & 8 \\
\hline
\end{tabular}

\section{RESULTS AND DISCUSSION}

The main performance measures utilized to measure the efficiency of MQTT and gateway performance are (throughput, end to end delay, packet send and packet receive).In this part, the comparison of the results of the scenarios carried out in the previous two experiments is made. The comparison is divided into three sections according to the evaluation criteria used.

\section{A. End to End Delay}

In the graph shown in Figure 11, the highest value of (8.92) sec was taken in the case of the normal gateway and compared with the eight ratios of the smart gateway. It was noted in the first ratio of smart 
(12.50\%) that, the value of the delay becomes (1.691) sec and in the second (25\%) became (2.791). The value of the delay continues to increase gradually with the proportion of urgent nodes until it reaches the eighth and the last case, which is (100\%) The value of the delay becomes equal or close to the delay value in the normal gateway. This shows us how successful the work of gateway is in reducing the ratio of the delay according to the packet rate in each ratio used and the signals to be passed according to the priority required.

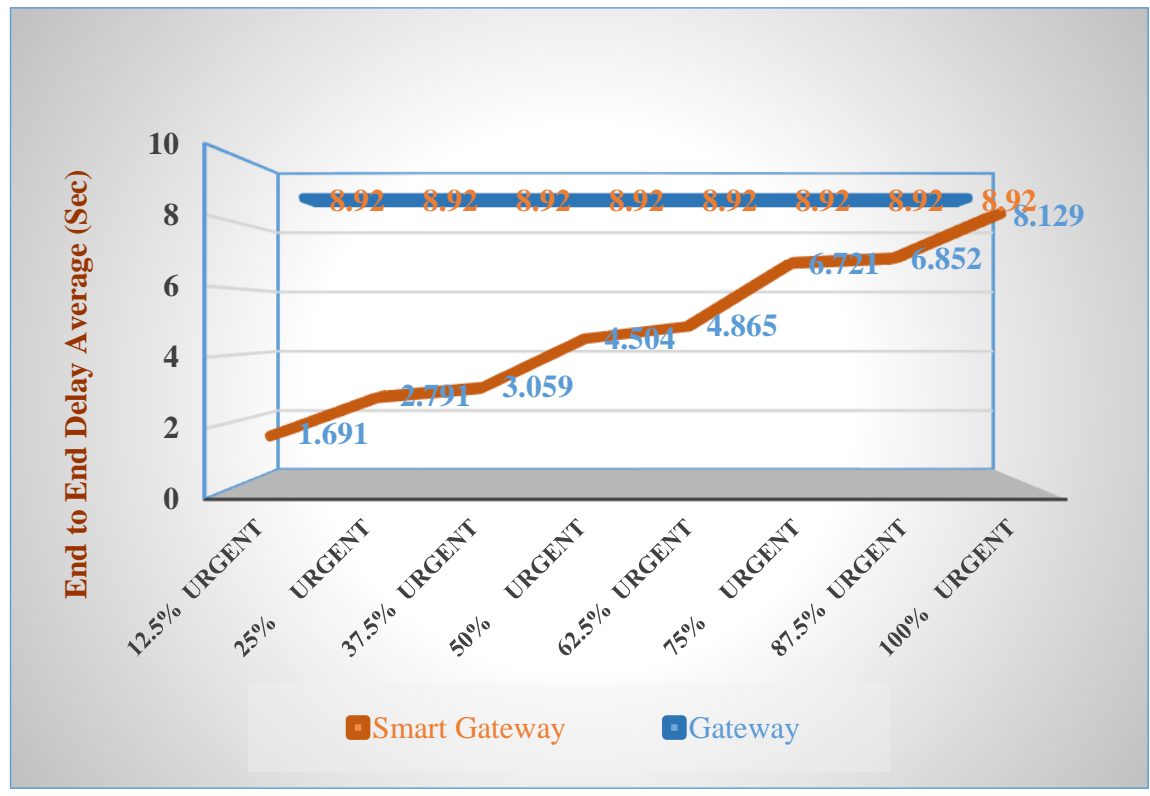

Fig.11. END to End DeLAY (SEC).

\section{B. Throughput}

The graph in Figure 12 shows that the highest throughput value of (68929.50) bit/sec was taken in the case of the normal gateway and compared with the eight urgent nodes ratios of the smart gateway. It was noted in the first-rate of (12.50\%) that, the value of throughput became (30141.092) bit/sec. In the second rate of (25\%) it became (36372.499) bit/sec. The value of throughput continues to increase gradually with the proportion of urgent nodes until it reaches the eighth, which is (100\%) that makes the throughput value reaches (73085.902) sec/bit. This illustrates how well the signal is transmitted and how smart the gateway is effectively applied in our work.

\section{Packet Sent and Received}

Through the graph is shown in Figure 13 and Figure 14 they show the comparison between the packets sent and received from the gateway to the broker. As we explained earlier in the case of delay and throughput by taking the highest value of the normal gateway and compared with the eight percentages of urgent nodes, where the proportions of urgent nodes for smart is gradually increasing until it reaches the rate of (100\%). In the case of the smart gateway, the eighth transmission rate has become higher than the normal gateway, due to the change that was made by applying the smart, but in the case of packet receipt, the values are very close or slightly higher at the smart gateway. 


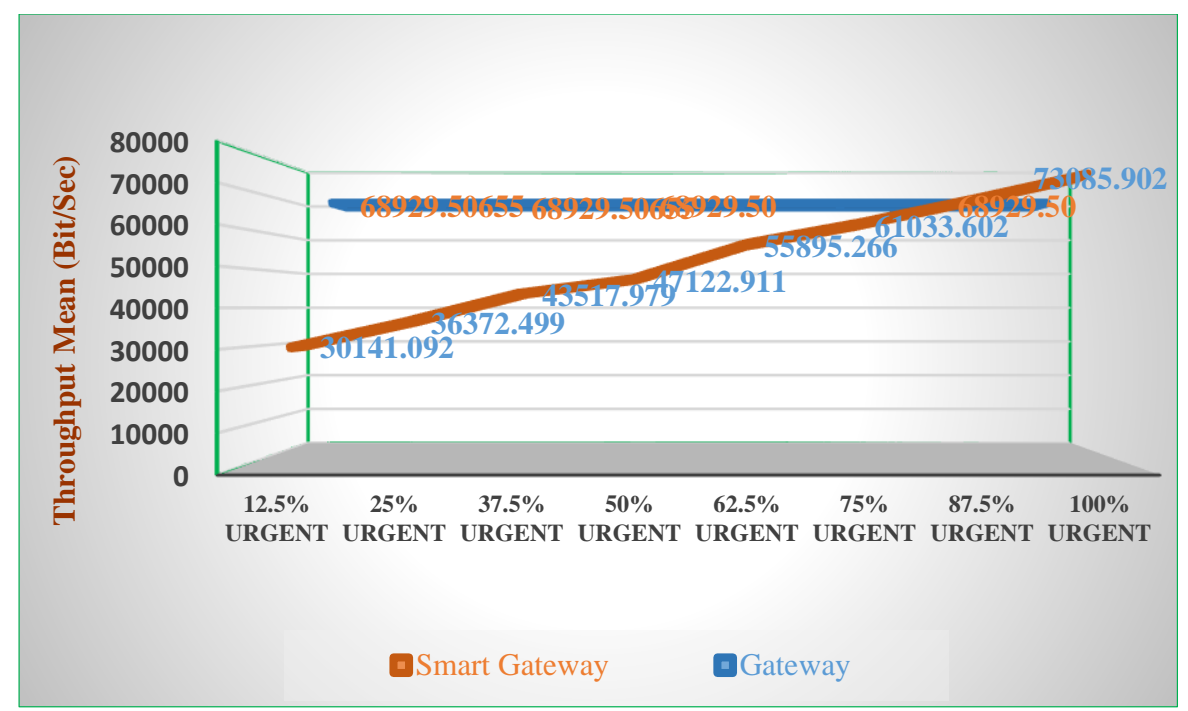

Fig.12. Throughrut (Bit/SeC).

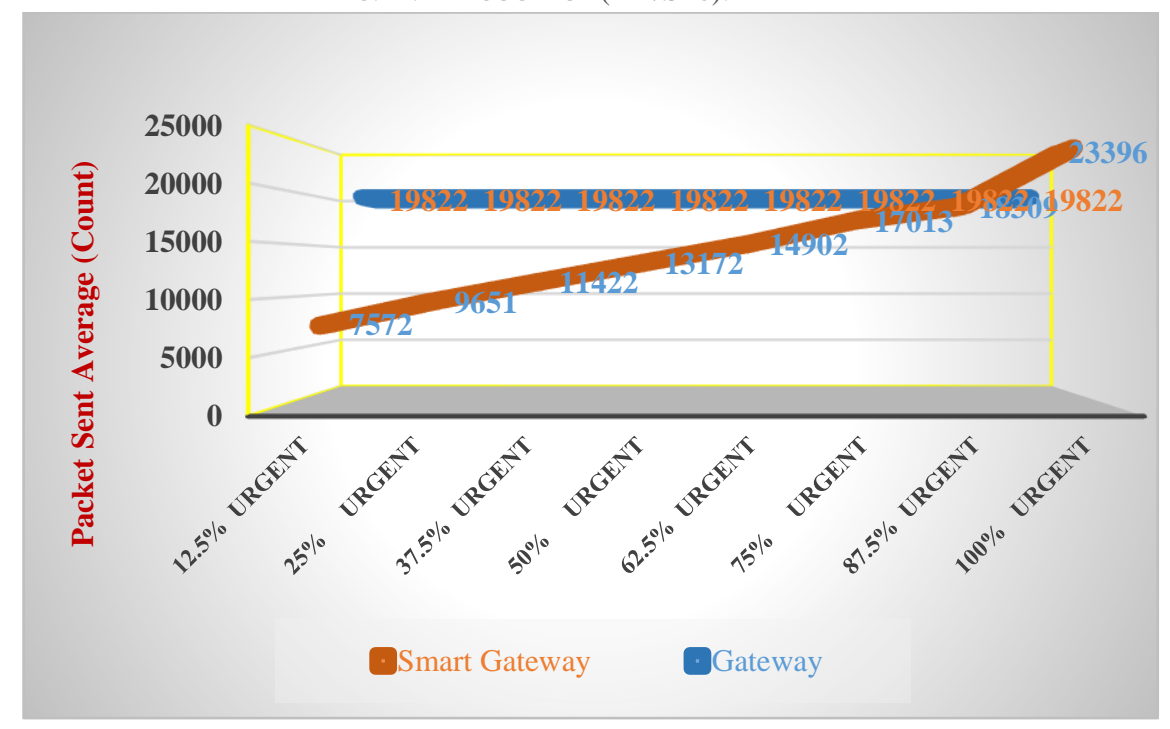

Fig.13. Packet Sent (Count).

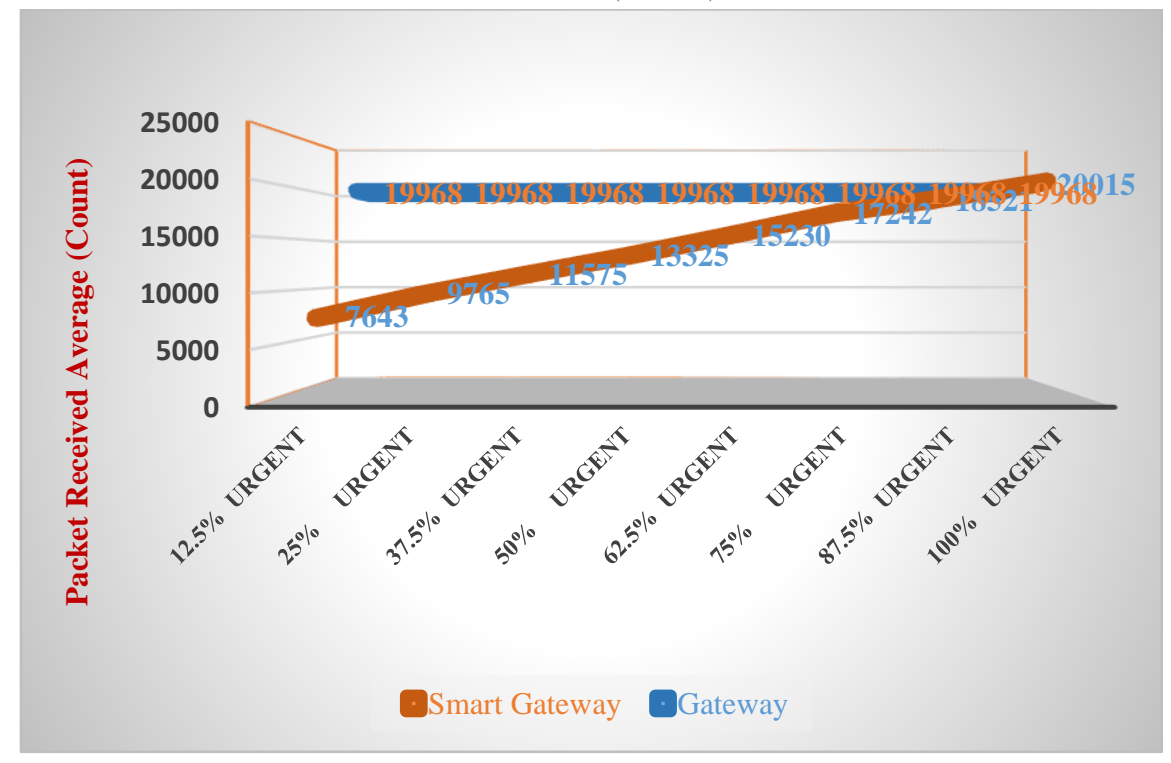

Fig. 14. Packet Received (Count). 


\section{CONCLUSION}

In this paper, the concept of the smart gate was introduced in health care. The gateway acts as a bridge to IP networks and cloud computing platforms for medical sensors and (home/hospital) building automation equipment. A new, simple and easy to use architecture has been implemented in which can be applied to any type of smart and that consisted of two main components: the local broker and the publisher/subscriber (Pub/Sub) models. The local broker works in the local network and model and the algorithm (Pub / Sub) is the main component and important in the construction of the gateway architecture and the importance of adding this model, highlights in conduct processing for data on any smart application before sending it. The principle of operation of this model is based on fog computing by processing the data coming from one side and then sent to another. The benefit of this smart gateway architecture is, where the Pub/Sub model is the one who adopts the work of smart by creating an application that has been called the smart apps. This application can contain many aspects of the smart and the most important:(i) Data compression, (ii) Artificial intelligence (Neural Network, Genetic Algorithm, etc.), (iii) Data filtering. The data filtering side has been taken and given it a priority from these many aspects, as an example in order to achieve the validation of the designed architecture of the gateway. Therefore, a test was performed using the ECG signal filtration application and prioritizing patients according to each patient's emergency. The cost of this architecture is very low because the using of added hardware is depending on the type of smart application that we want to use. This architecture may lead to a slight delay in the signal before sending it depends on the type of smart application in terms of the processor to be used. A remote ECG monitoring system based on the MQTT protocol has been proposed. The MQTT protocol is evaluated by two scenarios and the proposed design using the OMNET ++ simulation environment and its INET framework. In the framework of work, two experiments were conducted, the first experiment was for the gateway model, where the data rate of the transmitted packet was divided into three values of the ECG frequencies as shown in Table 1, conducting calculations and extracting the required results. The second experiment was for the smart gateway model, where the highest transmitting frequency value $(256 \mathrm{HZ})$ was taken which is only in urgent condition. Where this high value was distributed to eight relative cases as well as we conducted calculations and extracted the desired results. After comparing the two experiments, the results were observed in the urgent ratios for the eight cases of delay and throughput have gradually increased from the first case to the last case where the latter position is equal to the very high value of the sample rate used. Note that the delay in the first case became (1.691) sec, while the highest frequency value was (8.928) sec and the second case became (2.791) sec, and thus gradually increased until the eighth case (8.529) sec is equal to the highest frequency value. The same is true for throughput, packets sent and packets received as shown in Figures (12,13, and 14). This reduced the number of data sent as well as reduces the error rate. These results proved the success of the architectural and algorithm work that was implemented in the system so the smart gateway gave us many benefits at work and this is the goal of the project.

\section{REFERENCES}

[1] M. Houimli, "Formal Specification, Verification and Evaluation of the MQTT Protocol in the Internet of Things," 2017.

[2] A. Al-Fuqaha, "AL-FA-Internet of Things: A Survey on Enabling Technologies, Protocols, and Applications," IEEE Commun. Surv. Tutorials (Accepted Publ., vol. 1, no. 2, pp. 78-95, 2013.

[3] N. Pathania, "Traffic Prioritization in an MQTT Gateway," vol. 164, no. 2, pp. 32-38, 2017.

[4] A. Rahmani, N. K. Thanigaivelan, T. N. Gia, J. Granados, and B. Negash, "Smart e-Health Gateway: Bringing Intelligence to Internet-of-Things Based Ubiquitous Healthcare Systems," no. June, 2015.

[5] P. Santos et al., "A Pervasive System Architecture for Smart Environments in Internet of Things Context,” ICMI 2015 XIII Int. Conf. Multimodal Interact. London, UK, no. JANUARY, 2015. 
[6] Y. Lee and S. Nair, “A Smart Gateway Framework for IOT Services,” pp. 107-114, 2016.

[7] P. Karhula, "Internet of Things : a gateway centric solution for providing IoT connectivity," 2016.

[8] Z. Yang, Q. Zhou, L. Lei, K. Zheng, and W. Xiang, "An IoT-cloud Based Wearable ECG Monitoring System for Smart Healthcare,” J. Med. Syst., vol. 40, no. 12, 2016.

[9] A. M. Rahmani, T. Nguyen, B. Negash, and A. Anzanpour, "Exploiting smart e-Health gateways at the edge of healthcare Internet-of-Things: A fog computing approach," Futur. Gener. Comput. Syst., vol. 78, pp. 641-658, 2018.

[10] A. Mishra, A. Kumari, P. Sajit, P. Pandey, and A. Professor, "Remote web based ECG Monitoring using MQTT Protocol for IoT in Healthcare Internet of Things View project REMOTE WEB BASED ECG MONITORING USING MQTT PROTOCOL FOR IOT IN HEALTHCARE," Int. J. Adv. Eng. Res. Dev. Sci. J. Impact Factor (SJIF, vol. 571, no. 5, pp. 2348-4470, 2018.

[11] Q. Duy, M. V Ngo, T. Quang, T. Q. S. Quek, and H. Shin, "Enabling intelligence in fog computing to achieve energy and latency reduction," Digit. Commun. Networks, no. April, 2018.

[12] S. B. Kenitar, S. Marouane, A. Mounir, A. Younes, and A. G. Gonzalez, "Evaluation of the MQTT Protocol Latency over Different Gateways," pp. 1-5, 2018.

[13] L. Välimaa, "MQTT CLIENT IMPLEMENTATION IN IEC 61131-3 COMPATIBLE PROGRAMMING ENVIRONMENT,” no. January, 2017.

[14] K. Chen, W. S. Lu, F. Xue, L. Z. Zheng, and D. D. Liu, "Smart Gateway for Bridging BIM and Building," 2018.

[15] H. M. Hasan and B. K. Mohammed, "Evaluation of MQTT Protocol for IoT Based Industrial Automation," Int. J. Eng. Sci. Compute., no. December, 2018.

[16] R. Karthik, "ECG SIMULATION USING MATLAB Principle of Fourier Series."

[17] Omer Mukhtar wani, "Signal Processing of Stress Test ECG Using MATLAB," Int. J. Eng. Res., vol. V6, no. 08, pp. 175-183, 2017.

[18] D. Soni and A. Makwana, "A SURVEY ON MQTT A PROTOCOL OF INTERNET OF

THINGS(IOT),” no. April, 2017. 